\title{
A Case Report of Symptomatic Subclavius Posticus Muscle in a Synchronized Swimmer
}

\author{
Onur Levent Ulusoy, ${ }_{1}^{1}$ Burcin Sezgi Barlas, ${ }^{1}$ Ayhan Mutlu, ${ }^{1}$ Deniz Alis,,${ }^{2, *}$ and Meric Enercan ${ }^{3}$ \\ ${ }^{1}$ Department of Radiology, Istanbul Florence Nightingale Hospital, Istanbul, Turkey \\ ${ }^{2}$ Department of Radiology, Cerrahpasa Medical Faculty, Istanbul University, Istanbul, Turkey \\ ${ }^{3}$ Department of Orthopedics and Spine Surgery, Istanbul Florence Nightingale Hospital, Istanbul, Turkey \\ "Corresponding author: Deniz Alis, Department of Radiology, Cerrahpasa Medical Faculty, Istanbul University, Istanbul, Turkey. Tel: +90-05364797429, Fax: \\ +90-212414300021313, E-mail: drdenizalis@gmail.com \\ Received 2017 December 04; Revised 2018 February 12; Accepted 2018 April 28.
}

\begin{abstract}
Brachial plexus magnetic resonance (MR) examination of an adult female synchronized swimmer, who was suffering from rightsided arm pain and weakness while making a stroke, showed an aberrant "subclavius posticus muscle" in the parascapular region on the right side. The muscle had an attachment anteriorly on the first rib and posteriorly on to the scapula. The belly of this aberrant muscle showed proximity to the brachial plexus. Presence of such an anomalous muscle has been recognized as a possible cause of neurovascular compression or thoracic outlet syndrome (TOS). Radiologists and surgeons should be familiar with this rare entity and consider it in the differential diagnosis of TOS.
\end{abstract}

Keywords: Subclavius Posticus, Anatomic Variant, Thoracic Outlet Syndrome, Magnetic Resonance Imaging

\section{Introduction}

The subclavius posticus muscle (SPM) is a rare accessory muscle, generally asymptomatic and noticed incidentally. Clinical significance of this muscle has been recognized as a possible cause of neurovascular entrapment as in some patients it may be symptomatic and lead to thoracic outlet syndrome (TOS) (1-4).

This muscle may cause problems secondary to sudden or repetitive movements or trauma. We herein present a case of a synchronized swimmer with symptomatic SPM causing neurogenic TOS, and we focus on the imaging aspects of the pathology. Hence, detection of this accessory muscle is very valuable in such a complex anatomic region and may be a diagnostic dilemma. We present this case with magnetic resonance (MR) images in three orthogonal planes. Additionally, to the best of our knowledge, symptomatic brachial plexus entrapment secondary to SPM in an athlete (synchronized swimmer) after an intensive sports activity has not been reported in the literature.

\section{Case Presentation}

A 22-year-old semi-professional synchronized female swimmer presented with pain and weakness in the right shoulder and arm. The symptoms had begun two years previously and progressively increased since then. The pain worsened during swimming, especially when she was making a stroke. On physical examination, there were no signs suggestive of a glenohumeral joint disorder. She reported paresthesia and pain in her right arm when the shoulder was in 90 degrees abduction and external rotation. The cervical MR examination did not reveal any abnormality such as spondyloarthrosis, disc herniation or nerve root compression. Electromyographic studies did not show any compression neuropathies. To investigate neurogenic TOS, contrast-enhanced MR imaging (MRI) of the brachial plexus was performed. The MRI revealed a fusiform soft tissue structure, iso-intense with adjacent muscles in all sequences without contrast enhancement, which was compressing the brachial plexus fibers laterally and superiorly. This structure was identified as SPM (Figures 1 and 2). The MRI did not demonstrate any scalene muscle pathology, cervical ribs, long C7 transverse process, or mass lesion in this region. There was no plexitis or neuritis detected as brachial plexus fibers did not show pathologic signal changes or contrast enhancement. Furthermore, there were no signal changes or atrophy of the muscles surrounding the scapula and shoulder joint suggesting denervation myopathy. Based on the clinical examination and MRI, the patient was diagnosed with neurogenic TOS caused by compression of the brachial plexus due to SPM. 

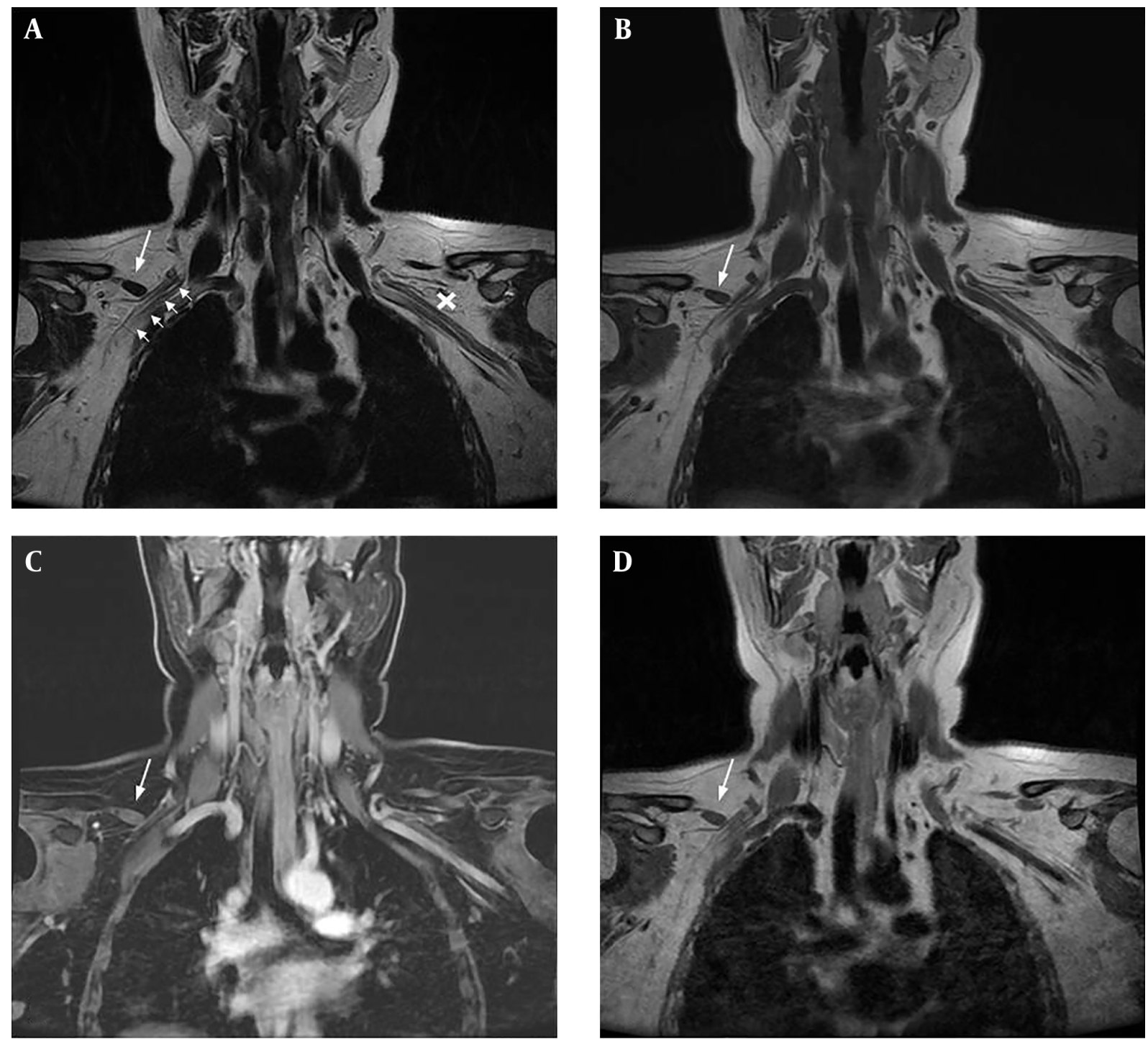

Figure 1. Brachial plexus MRI of a 22-year-old female with progressive pain and weakness in the right shoulder and arm from two years ago. A, Coronal T2-weighted image [repetition time (TR) $4170 \mathrm{~ms}$, echo time (TE) $80 \mathrm{~ms}$ ]; B, Coronal T1-weighted image (TR $400 \mathrm{~ms}$, TE $10 \mathrm{~ms}$ ); C, Contrast enhanced fat-suppressed coronal T1-weighted image (TR $290 \mathrm{~ms}$, TE $4 \mathrm{~ms}$ ); and D, Contrast enhanced coronal T1-weighted image (TR $400 \mathrm{~ms}$, TE $10 \mathrm{~ms}$ ). Ovoid structure, isointense with the muscle in all sequences without contrast enhancement (arrows in A-D), and slightly compressing the fibers of the right brachial plexus (short arrows in A) laterally and superiorly. There is no muscle that corresponds to the subclavius posticus muscle on the contralateral side ( $\operatorname{cross}$ in $\mathrm{A}$ )

Conservative management, instead of surgery, was utilized upon the request of the patient. The symptoms decreased significantly following oral analgesics, physical therapy to maintain the scapula in retraction/elevation and an exercise program. At the 6-month follow up, she was able to return to her training without any difficulties.

\section{Discussion}

TOS results from compression of the brachial plexus and subclavian vessels. Cervical ribs, long C7 transverse process, fibrous bands, scalene muscles abnormalities, accessory muscles, and mass lesions in this region may cause this symptomology (5). There are two types of TOS: vascular and neurogenic. True neurogenic TOS is mostly a diagnosis of exclusion, based upon physical examination and diagnostic tests made to investigate the differential diagnoses. The exacerbation of the symptoms with abduction and retraction maneuvers that tether the brachial plexus suggests a TOS. Vascular etiologies include arterial or venous compression with or without thrombosis.

SPM is an accessory muscle that courses between the 

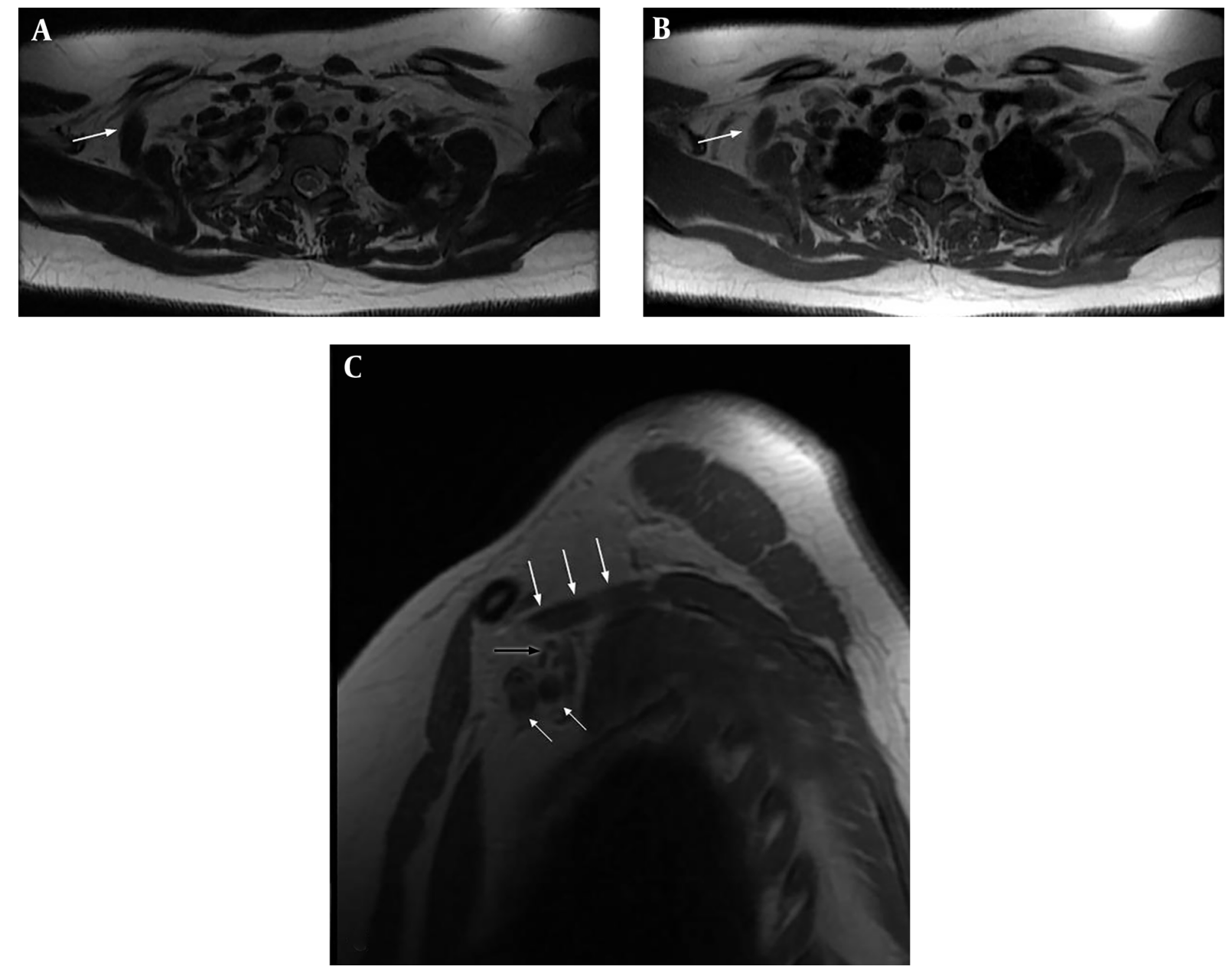

Figure 2. Brachial plexus MRI. A, Axial T2-weighted image [repetition time(TR) 4105 ms, echo time(TE) 120 ms]; B, Axial T1-weighted image(TR 410 ms, TE 12 ms); and C, Contrast enhanced sagittal T1-weighted image (TR $400 \mathrm{~ms}$, TE $12 \mathrm{~ms}$ ) show that the fusiform structure (white arrows in A-C) isointense with the muscle, showing close proximity to the superior trunk of the right brachial plexus (black arrow in C). Thin white arrows in C indicate subclavian artery and vein.

anterior aspect of the first rib and the upper medial border of the scapula (6-8). Inferiorly, the muscle is adjacent to the brachial plexus and the subclavian vessels. Two other variations at this level, the scapuloclavicularis (posterior to the coracoid process, between the transverse scapular ligament and the clavicle) and the pectoralis intermedius (traverses from the 3rd and 4th ribs to the coracoid process, between the pectoralis major and minor muscles) muscles are included in the SPM variation because they have a similar anatomic matrix and innervations (7). The incidence of SPM is $8.9 \%$ and in more than $90 \%$ cases it is unilateral (1). Bilateral cases have also been reported (9).

Although SPM is mostly asymptomatic, the anatomical relationships of this aberrant muscle with the brachial plexus and the subclavian vessels might arouse clinical significance, since it might lead to TOS in some cases. Smayra et al. (10) presented a case of SPM that caused TOS in a young woman. Forcada et al. (2) showed that it could even form a groove on the upper trunk of the brachial plexus. Muellner et al. (3) demonstrated that SPM, depending on its activation, may cause dynamic compression on the brachial plexus, and in their study, they showed this dynamic compression using MRI (arm abduction). SPM could also be a possible factor for venous TOS, known as Paget von-Schrötter syndrome, as it may cause thrombosis of the axillary and/or the subclavian vein due to the extrinsic compression (1).

This muscle may cause problems secondary to sudden or repetitive movements or trauma. Ciampi et al. (11) demonstrated bilateral SPM in two young overhead athletes who presented with pain and weakness in the dominant upper limb, and they established diagnosis using MR angiography. Ozcakar et al. (4) have reported a case of TOS after a traction injury and demonstrated an aber- 
rant SPM compressing brachial plexus using MRI. Surgery via transaxillary approach was performed, and SPM was resected. Cogar et al. (12) reported another case with symptomatic SPM after trauma. In their case, an adult male triathlon athlete was suffering from symptoms of suprascapular nerve entrapment for five months after a fall from his bicycle. MRI identified a SPM surrounding suprascapular nerve and after surgical excision of the SPM, his symptoms showed rapid resolution. In our case, SPM was asymptomatic, but after an intensive exercise program, it became symptomatic gradually. Our patient's symptoms resolved after a modification in her workout program and conservative physical treatment. Regarding the etiology, we suggested brachial plexus entrapment secondary to SPM after an intensive sports activity is likely as relevant as the previously reported case of suprascapular nerve entrapment secondary to SPM after trauma in an athlete (12).

Patients with a suspicion of TOS are evaluated with cross-sectional studies such as computed tomography(CT) or MRI (13). Radiologic studies are valuable in the exclusion of common etiologies. MRI has a pivotal role in determining the type of TOS. In addition to the assessment of the brachial plexus, MRI provides information on the surrounding soft tissues, bone, and muscle. Advanced studies, including MR angiography, MR venography, CT angiography and digital subtraction angiography may be used to exclude venous and arterial TOS. An abnormal spaceoccupying structure with proximity to the brachial plexus or compressing it, and the paucity of fatty tissues around the plexus on MRI are suggestive of neurogenic TOS. It may or may not cause pressure neuritis, which is detected based on the presence of brachial plexus edema or enhancement. A band of non-enhancing tissue stretching from the first costal cartilage to the superior angle of the scapula, isointense to the adjacent muscles, is the classic feature of the SPM on MRI $(3,4,12)$.

Management of TOS relies on decreasing factors that exacerbate the symptoms (9). The first option on the treatment of TOS secondary to SPM is conservative management (8). Surgical procedures may be warranted in cases that are unresponsive to conservative treatment or may even be considered as the first choice in the presence of severe neurologic deficits $(4,10)$.

In conclusion, SPM is a rare accessory muscle, which is mostly asymptomatic. Impingement on the brachial plexus or subclavian vasculature due to trauma or overuse of this muscle may lead to TOS. Radiologists and surgeons should be familiar with this rare entity and consider it in the differential diagnosis of TOS. High-resolution MRI is an essential adjunct to anamnesis and physical examination in the diagnosis.

\section{Footnote}

Conflict of Interest: The authors declare that they have no conflict of interest.

\section{References}

1. Akita K, Ibukuro K, Yamaguchi K, Heima S, Sato T. The subclavius posticus muscle: a factor in arterial, venous or brachial plexus compression? Surg Radiol Anat. 2000;22(2):111-5. [PubMed: 10959678].

2. Forcada P, Rodriguez-Niedenfhr M, Llusa M, Carrera A. Subclavius posticus muscle: supernumerary muscle as a potential cause for thoracic outlet syndrome. Clin Anat. 2001;14:55-7.

3. Muellner J, Kaelin-Lang A, Pfeiffer O, El-Koussy MM. Neurogenic thoracic outlet syndrome due to subclavius posticus muscle with dynamic brachial plexus compression: a case report. BMC Res Notes. 2015;8:351. doi: 10.1186/s13104-015-1317-3. [PubMed: 26268814]. [PubMed Central: PMC4535788]

4. Ozcakar L, Guney MS, Ozdag F, Alay S, Kiralp MZ, Gorur R, et al. A sledgehammer on the brachial plexus: thoracic outlet syndrome, subclavius posticus muscle, and traction in aggregate. Arch Phys Med Rehabil. 2010;91(4):656-8. doi: 10.1016/j.apmr.2010.01.001. [PubMed: 20382302].

5. Pang D, Wessel HB. Thoracic outlet syndrome. Neurosurgery. 1988;22(1 Pt 1):105-21. [PubMed: 3278249].

6. Gupta G, Prakash S, Ojha P. A case of bilateral subclavius posticus muscle.J Evolut Med Dental Sci. 2013;2:761-4.

7. Sarikcioglu L, Sindel M. A case with subclavius posticus muscle. Folia Morphol (Warsz). 2001;60:229-31.

8. Singhal S, Rao VV, Manjunath KY. Subclavius posticus muscle - A case report. Int J Morphol. 2008;26:813-5.

9. Martin RM, Vyas NM, Sedlmayr JC, Wisco JJ. Bilateral variation of subclavius muscle resembling subclavius posticus. Surg Radiol Anat 2008;30(2):171-4. doi: 10.1007/s00276-008-0303-z. [PubMed: 18231702].

10. Smayra T, Nabhane L, Tabet G, Menassa-Moussa L, Hachem K, HaddadZebouni S. The subclavius posticus muscle: an unusual cause of thoracic outlet syndrome. Surg Radiol Anat. 2014;36(7):725-8. doi: 10.1007/s00276-013-1227-9. [PubMed: 24166072].

11. Ciampi P, Agnoletto M, Scotti C, Ballis R, Gerevini S, Peretti GM, et al. Thoracic Outlet Syndrome in the Overhead Athlete: A Report of 2 Cases of Subclavius Posticus Muscle. Clin J Sport Med. 2017;27(3):e29-31 doi: 10.1097/JSM.0000000000000329. [PubMed: 27379658].

12. Cogar AC, Johnsen PH, Potter HG, Wolfe SW. Subclavius posticus: an anomalous muscle in association with suprascapular nerve compression in an athlete. Hand (N Y). 2015;10(1):76-9. doi: 10.1007/s11552-0149652-8. [PubMed: 25762884]. [PubMed Central: PMC4349834].

13. Aralasmak A, Karaali K, Cevikol C, Uysal H, Senol U. MR imaging findings in brachial plexopathy with thoracic outlet syndrome. AJNR Am J Neuroradiol. 2010;31(3):410-7. doi: 10.3174/ajnr.A1700. [PubMed: 19815618]. 